\title{
Folate metabolism abnormalities in autism: potential biomarkers
}

\author{
Richard E Frye* ${ }^{*}$, John C Slattery ${ }^{1}$ \& Edward V Quadros ${ }^{2}$ \\ ${ }^{1}$ Autism Research Program, Arkansas Children's Research Institute, Little Rock, AR 72202, USA \\ ${ }^{2}$ Department of Medicine, State University of New York - Downstate Medical Center, Brooklyn, NY 11203, USA \\ * Author for correspondence: Tel.: +1 501364 1850; Fax: +1 501978 6483, REFrye@uams.edu
}

\begin{abstract}
Autism spectrum disorder (ASD) has been linked to abnormalities in folate metabolism. Polymorphisms in folate genes may act in complex polygenic ways to increase the risk of developing ASD. Autoantibodies that block folate transport into the brain have been associated with ASD and children with ASD and these autoantibodies respond to high doses of a reduced form of folate known as folinic acid (leucovorin calcium). Some of the same abnormalities are also found in mothers of children with ASD and supplementing folate during preconception and gestational periods reduces the risk to the offspring from developing ASD. These data suggest that folate pathway abnormalities may be a major metabolic disturbance underlying ASD that can be leveraged as biomarkers to improve symptoms and prevent ASD.
\end{abstract}

First draft submitted: 29 March 2017; Accepted for publication: 23 May 2017; Published online: 3 August 2017

Keywords: autism spectrum disorder $\bullet$ cerebral folate deficiency $\bullet$ folate $\bullet$ folate receptor $\alpha$ autoantibodies - folinic acid • genetic-environmental interactions • methylation • microbiome • mitochondria • prenatal folate supplementation

\section{Autism spectrum disorder: an emerging disorder with unclear etiology}

Autism spectrum disorder (ASD) is a neurodevelopmental disorder that affects about $2 \%$ of children in the USA [1]. Although it is defined through observation of the presence of abnormal behavior and absence of typical developmental milestones, ASD is associated with a wide range of medical abnormalities such as sleep, immune and gastrointestinal disorders and epilepsy. In addition to medical comorbidities, many children with ASD have disruptions in body physiology such as abnormalities in folate, cobalamin, tetrahydrobiopterin $\left(\mathrm{BH}_{4}\right)$, neurotransmitter, carnitine, redox and mitochondrial metabolism [2]. The connection between underlying physiological abnormalities and behavior is an area of active research. In this review, we will provide evidence that abnormalities of one-carbon folate metabolism are particularly important in ASD.

Although the etiology of ASD is not known, genetics has been the focus of ASD research for many decades [3]. A notion of a pure genetic etiology is tempered by the fact that single gene and chromosomal defects are found in a minority of ASD cases, accounting for only $15.8 \%$ of cases when both chromosomal microarray and whole exome sequencing were used in one recent large study [4]. ASD has also been associated with exposures to numerous environmental agents during the prenatal and postnatal period [5]. Studies that have examined the contribution of the environmental and genetic components highlight that ASD arises as a consequence of complicated interactions between genetic predisposition and environmental factors [6,7]. As we will outline in this review, abnormalities in folate metabolism provide an excellent model of how environment factors can interact with genetic predisposition to cause ASD.

At this time, the standard of care for ASD treatment is applied behavioral analysis and other equivalent behavioral interventions along with educational, developmental, occupational and speech therapy. Optimal application of behavioral therapy requires full-time one-on-one therapist for many years; however, even when this optimal therapy is applied, suboptimal outcomes are common. In addition, controlled studies examining the efficacy of various behavioral therapies are lacking [8]. The identification of physiological biomarkers that can help identify core biological processes that are amenable to treatment are urgently needed. Ideally such an approach would be 
Table 1. Recommended dietary allowance and upper limit in dietary folate equivalents.

\begin{tabular}{|lll|}
\hline Age $/$ gender & Recommended dietary allowance & Upper limit \\
\hline $0-6$ months & $65 \mathrm{mcg}$ & \\
\hline $7-12$ months & $80 \mathrm{mcg}$ & $300 \mathrm{mcg}$ \\
\hline $1-3$ years old & $150 \mathrm{mcg}$ & $400 \mathrm{mcg}$ \\
\hline $4-8$ years old & $200 \mathrm{mcg}$ & $600 \mathrm{mcg}$ \\
\hline $9-13$ years old & $300 \mathrm{mcg}$ & $800 \mathrm{mcg}$ \\
\hline $14-18$ years old & $400 \mathrm{mcg}$ & $1000 \mathrm{mcg}$ \\
\hline Typical adult males & $400 \mathrm{mcg}$ & $1000 \mathrm{mcg}$ \\
\hline Typical adult females & $400 \mathrm{mcg}$ & $800 \mathrm{mcg}$ \\
\hline Pregnancy $(14-18$ years old) & $600 \mathrm{mcg}$ & $1000 \mathrm{mcg}$ \\
\hline Pregnancy $(>18$ years old $)$ & $600 \mathrm{mcg}$ & $800 \mathrm{mcg}$ \\
\hline Lactating $(14-18$ years old $)$ & $500 \mathrm{mcg}$ & $1000 \mathrm{mcg}$ \\
\hline Lactating $(>18$ years old $)$ & $500 \mathrm{mcg}$ & \\
\hline
\end{tabular}

Table 2. Polymorphisms in folate genes related to autism spectrum disorder.

\begin{tabular}{|c|c|}
\hline Folate Gene & Variation \\
\hline PCFT & Not studied \\
\hline RFC & $80 \mathrm{G}>\mathrm{A}$ \\
\hline MRP3 & Not studied \\
\hline GGH & Not studied \\
\hline DHFR & 19 bp-deletion \\
\hline MTHFR & C667T, A1298C \\
\hline FOLR1 & Not studied \\
\hline
\end{tabular}

DHFR: Dihydrofolate reductase; GGH: Gammaglutamyl hydrolase; MTHFR: Methylenetetrahydrofolate reductase; PCFT: Proton-coupled folate transporter; RFC: Reduced folate carrier

combined with behavioral therapies to synergistically improve ASD symptoms. To this end, we will outline how abnormalities in folate metabolism can be targeted in order to attempt to achieve this goal.

\section{Dietary folate}

Folate, an essential vitamin, must be derived from the diet since the body cannot produce it de novo. Food rich in folate includes leafy vegetables (foliage), beans, nuts and meats. The importance of folate is so widely recognized that many foods are fortified with folic acid, the inactive oxidized form of folate, in modern western society. Folic acid is prominent in multivitamins, particularly prenatal multivitamins. Recommended dietary allowance (average daily intake sufficient for most people) for folate varies with both age and gender and is given in dietary folate equivalents to account for the fact that folic acid is less bioavailable in food and when supplements are taken with food (Table 1) [9]. In general, folic acid in food is about $50 \%$ bioavailable. Although there are no known adverse effects of excess folate intake, there is some speculation that high folate intake could accelerate precancer cells that already exist since folate is a key nutrient for rapidly dividing and proliferating cells; however there are no empirical data to support this notion and folates are widely recognized to have excellent long-term safety profile. Nevertheless, upper limits for daily folate intake have been established.

\section{The importance of the microbiome $\&$ folate transporters in folate absorption}

Folate is absorbed in the GI tract by three transporters (Figure 1). The proton-coupled folate transporter (PCFT) is located in the upper part of the intestine and operates best in acidic conditions. One important consideration is that many children with ASD are on medication such as proton pump inhibitors because of gastrointestinal symptoms. Such medications may decrease acidity in the proximal gut, potentially leading to decreased function of the PCFT. Studies in adults suggest that proton pump inhibitors do not significantly change blood folate levels [10] but such studies have not been conducted in children with ASD. The reduced folate carrier (RFC) is responsible for absorbing folate in the lower intestinal tract and the 80G $>$ A RFC polymorphism has been associated with increased ASD risk [11] (Table 2). The PCFT and RFC facilitate folate entering the enterocyte in the intestinal 


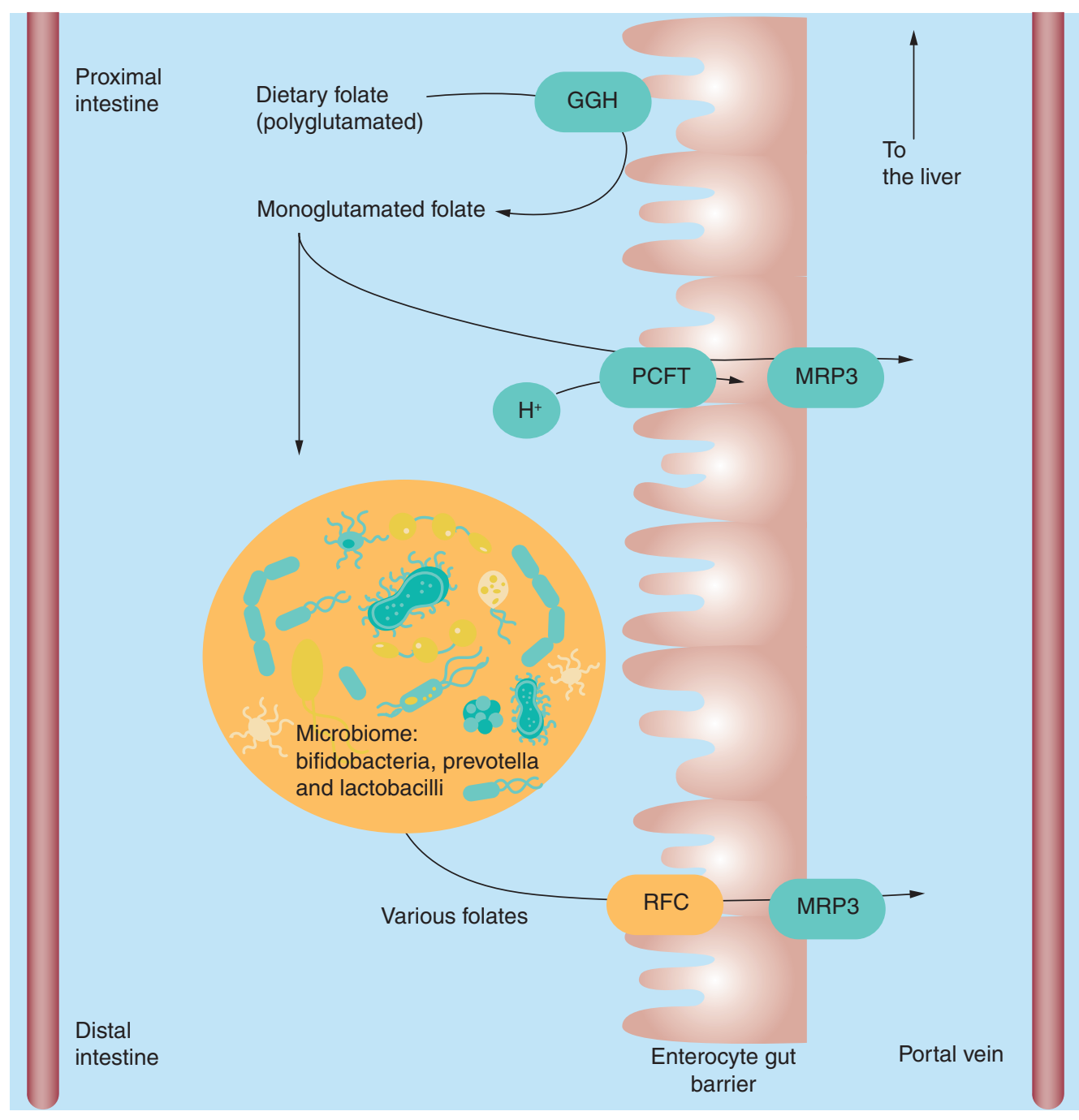

Figure 1. Transportation of folate into the body from the gut. Items shaded in orange are known to be abnormal in individuals with autism spectrum disorder.

GGH: Gammaglutamyl hydrolase; PCFT: Proton-coupled folate transporter; RFC: Reduced folate carrier.

lining while the multidrug resistance associated protein 3 transports folate out of the enterocyte and into the portal vein. Although both PCFT and multidrug resistance associated protein 3 genes have known variations, variations in these genes have not been studied in ASD.

The enteric microbiome, a diverse ecosystem of bacteria, fungi, viruses and their collective genomes, resides in the GI tract and aids in vitamin synthesis, immune regulation and host metabolism [12]. Folate is synthesized by enteric bacterial species, particularly Bifidobacteria, Prevotella and certain Lactobacilli species [13-17]. Children with ASD lack microbial diversity and have a decreased abundance in both Prevotella and Bifidobacteria, potentially leading to reduced folate production by microbiota in individuals with ASD [18]. Deficiencies in these enteric bacteria could be linked to ASD through environmental factors. Of note, Bifidobacteria is primarily obtained through breastfeeding and reduced breastfeeding has been linked to ASD, and western diets, which have been linked to ASD, are low in Prevotella [18]. A recent small open-label clinical trial showed that microbiota transfer therapy significantly improved gastrointestinal and ASD symptoms in children and adolescents with ASD [16]. Interestingly, two of the primary species that showed significant increases in abundance were Bifidobacteria and Prevotella, raising the question of whether an improvement in folate status accounted for some of the clinical effects in this study. 


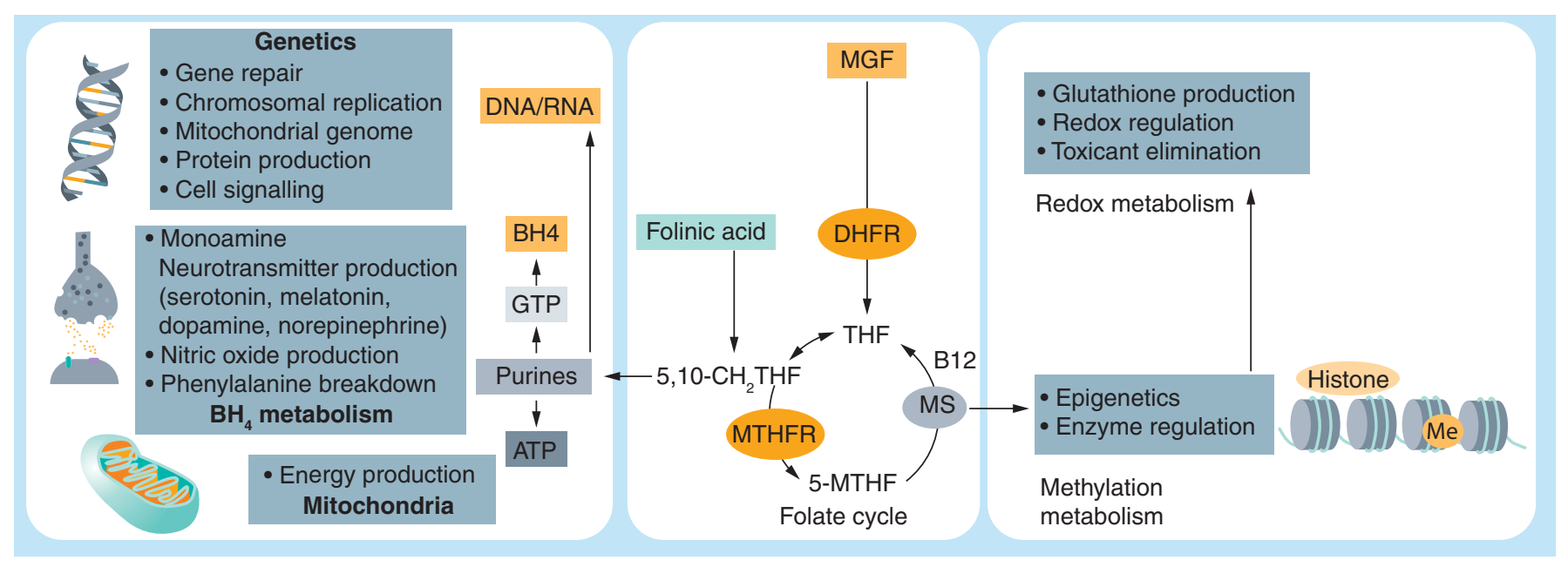

Figure 2. The folate cycle and its connection with other critical metabolic processes. In this diagram important cellular metabolites are featured in boxes while ovals outline critical metabolic enzymes. Those shaded in orange are metabolites and enzymes that are known to be abnormal in individuals with autism.

DHFR: Dihydrofolate reductase; MGF: monoglutamated folate; MS: Methionine synthase; MTHFR: Methylenetetrahydrofolate reductase; THF: Tetrahydrofolate.

\section{Activation of folate $\&$ the folate cycle}

The main circulating plasma form of folate is 5-methyltetrahydrofolate (5-MTHF). The monoglutamated folate (MGF) and folic acid derived from the diet is converted into tetrahydrofolate (THF) using the dihydrofolate reductase (DHFR) enzyme that converts folate derivatives to dihydrofolate and then to THF (Figure 2). This reaction requires niacin (vitamin B3) and can be inhibited by certain medications including antibiotics and certain cancer treatments, specifically methotrexate, trimethoprim and pyrimethamine. Polymorphisms in DHFR that have been linked to ASD can decrease the efficiency of this enzyme (Table 2) [19]. THF is the substrate for the production of formyl, methyl and methylene forms of folate. The latter is converted to 5-MTHF by the enzyme methylenetetrahydrofolate reductase (MTHFR). Several common polymorphisms can decrease the efficiency of MTHFR enzyme function. Several of these polymorphisms, particularly the C667T and A1298C, are more common in individuals with ASD as compared with the typically developing population [20]. Other folates, such as folinic acid, can enter the folate cycle at various points (Figure 2).

\section{Folate, one of the body's most important vitamins}

Folate is critical for physiologic systems associated with neurodevelopment (Figure 2). Folate is essential for de novo purine synthesis [21]. Purines are nucleic acid building blocks of DNA and RNA. When cells divide and replicate, they need to copy large amounts of DNA. Thus, folate is extremely important during gestation when the embryo develops and rapidly grows as well as during early life when the body is quickly growing. Insufficient purines can result in errors in DNA replication resulting in mutation and chromosomal abnormalities. Purines are also essential for producing RNA. RNA carries the genetic message from the nucleus into the cytoplasm to produce proteins and enzymes. In addition, research over the past decade has highlighted the important role of RNA in the regulation of molecular pathways through mRNAs and research studies have confirmed that folate deficiency can alter miRNA expression [22].

Guanosine is the purine that is essential for producing GTP, a precursor of $\mathrm{BH}_{4}[23,24] . \mathrm{BH}_{4}$ is essential for producing many important molecules for cellular regulation and communication, including nitric oxide and the monoamine neurotransmitters [23,24]. Nitric oxide, critical for blood flow regulation, is involved in the immune response and is believed to be a novel neurotransmitter. Production of the monoamine neurotransmitters dopamine, serotonin and norepinephrine as well as the circadian regulator melatonin, all require $\mathrm{BH}_{4}$. Interestingly, abnormalities in all of these pathways have been associated with ASD [23].

Adenosine is another important purine that is the precursor to ATP, the energy carrier of the cell and is essential for mitochondrial function. ATP not only carriers the energy produced by the mitochondria but also regulates 
mitochondrial function. Thus, the availability of ATP is essential for cellular metabolism and it has recently been demonstrated that folate supplementation may significantly improve mitochondrial function [25].

Folate is critical for the production of S-adenosylmethionine, the major methyl donor, from methionine [26]. Methionine is produced by methionine synthase, a cobalamin (vitamin B12) dependent enzyme that also converts 5-MTHF to THF. Methylation is essential for regulating chromosomal dynamics, the expression of genes and the function of enzymes. Methylation is connected to redox metabolism because homocysteine is an upstream precursor to glutathione, the body's major intrinsic antioxidant. Glutathione is essential for detoxification and enzyme regulation [26]. Oral low-dose folinic acid given concurrently with subcutaneously injected methylcobalamin increases glutathione production and improves development in children with ASD [27].

\section{Animal models of the effects of folate deficiency on development}

To better understand the consequences of folate on neurodevelopment, folate-deficient rodents have been studied. Pups born to rats placed on a folate-deficient diet have significantly decreased brain mass and myelin [28] as well as behavioral and functional abnormalities [28-30]. In a mouse model of maternal folate deficiency, premature differentiation of the dorsal root ganglion followed by a reduction in cell number and disruption in architecture was observed [31]. Suboptimal folate deficiency in dams during pregnancy can produce behavioral disorders in pups even after their folate status is fully restored. These disorders can manifest in the form of anxiety-related behavior [32].

Folate deficiency early in life can also have profound effects on the developing brain. Folate deficiency during weaning induced memory and cognitive deficits in rats, including spatial learning, long-term memory and setshifting deficits [28]. These effects of folate deficiency can also be produced in older rats, as demonstrated in 6-month-old male rats fed a folate-deficient diet for 8 weeks [33]. Electron microscopy of the brain in these rats showed degenerative changes in the cerebral microvasculature [33]. Furthermore, folate plays an important role in axonal regeneration and repair after induced brain damage in rodent models [34].

\section{Folate deficiency can be isolated to the brain}

About 15 years ago, clinical researchers described a case series of children with normal neurodevelopment during early infancy followed by neurodevelopmental regression along with the development of irritability, insomnia and pyramidal tract signs. Some children developed epilepsy and movement disorders, such as dyskinesia, choreoathetosis and ataxia. Their head circumferences also stopped growing normally resulting in acquired microcephaly and MRI demonstrated brain atrophy and delayed myelination. Some older children also demonstrated hearing and vision loss. What was unique about these children is that they all had low concentrations of 5-MTHF in their cerebrospinal fluid (CSF) but normal 5-MTHF concentrations in their blood [35]. Since CSF is representative of folate concentrations in the brain, this newly described neurodevelopmental disorder was called cerebral folate deficiency (CFD) syndrome to signify deficiency of folate specific to the brain.

\section{Folate transporters}

Once folate is in the body, it is transported into tissues using several mechanisms. In addition to the PCFT and $\mathrm{RFC}$, there is the folate receptor (FR) mechanism for transporting folate. There are four FRs: $\alpha, \beta, \gamma$ and $\delta$. Here we will concentrate on the FR-alpha (FR $\alpha)$ because of its importance in neurodevelopment and CFD.

Folate crosses the blood-brain barrier using two transporter mechanisms located at the choroid plexus (Figure 3). The FR $\alpha$-mediated transport is the primary transport mechanism because it has a high affinity for folate. Using this mechanism, folate binds to the FR $\alpha$ on the apical side of the cell and the receptor undergoes endocytosis and is transported to the basal side of the cell where it is then released into the brain. This is an active transport mechanism that requires ATP. The RFC acts as an alternate system to transport folate into the brain. However, it is important to note that the RFC is not as efficient at transporting folate as the FR $\alpha$ mechanism primarily because it has lower affinity for folate compared with the FR $\alpha$. However, the RFC has a critical role in the CNS since it is the primary carrier of folate into neurons.

Folate transport into the fetus is similar to folate transport into the brain. The FR $\alpha$, RFC and PCFT are important for transporting folate into the syncytiotrophoblast cells while RFC is important for transporting folate from the syncytiotrophoblast cells into the cytotrophoblast cells and onward into the fetal blood (Figure 3). However, current evidence suggests that FR $\alpha$ is the primary transporter because blocking this transporter with an antibody significantly decreases folate transport to the fetus. 


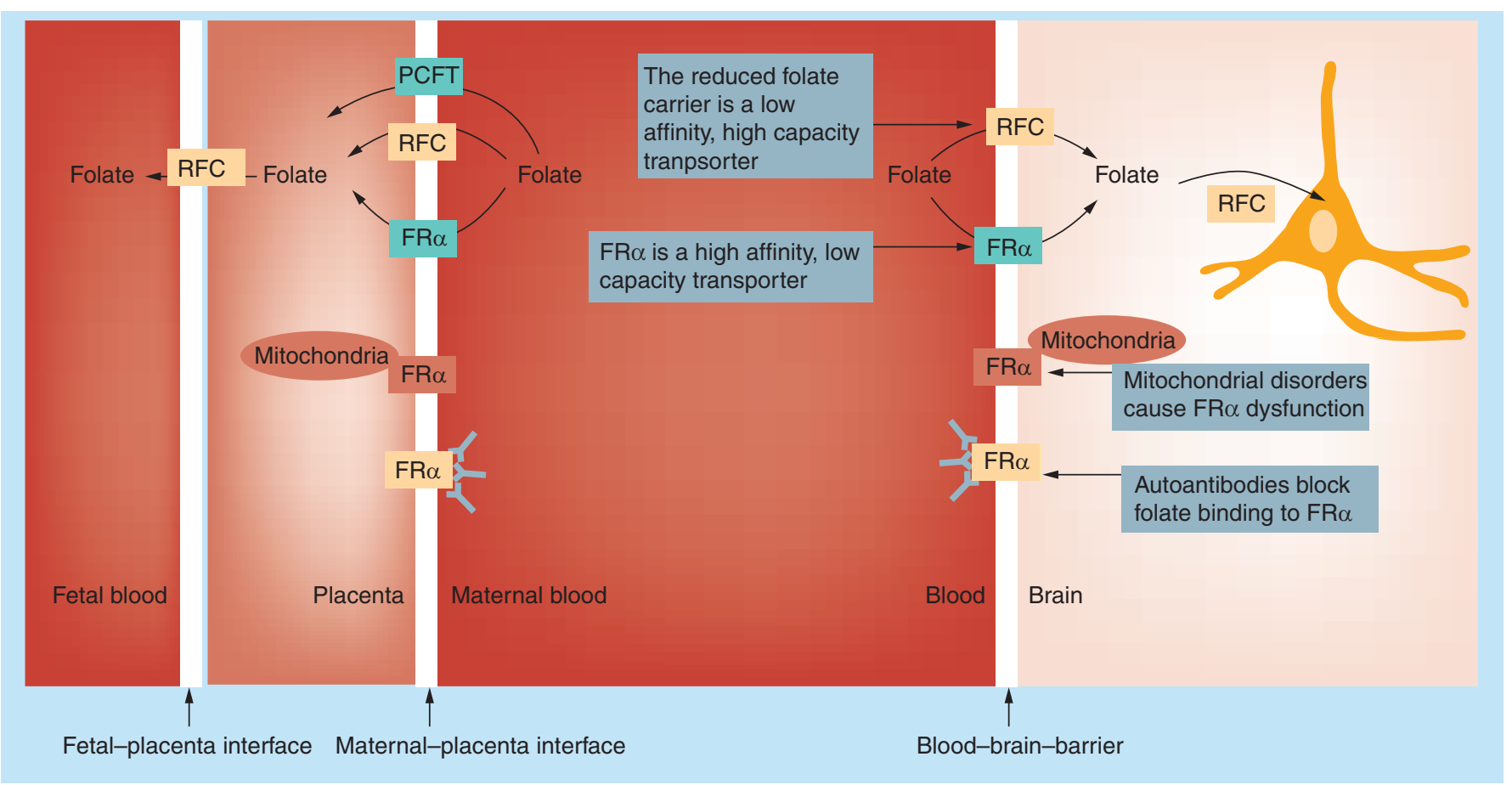

Figure 3. Folate transport to the fetus and into the brain. Those shaded in orange are metabolites and enzymes that are known to be abnormal in individuals with autism. Highlighted in red signifies the presence of dysfunctional energy transport; and effects on folate transport are highlighted.

FR: Folate receptor; PCFT: Proton-coupled folate transporter; RFC: Reduced folate carrier.

\section{CFD: discovering the cause}

At the time CFD was described, the cause of CFD was not known. Since the brain's main folate transporter is the FR $\alpha$, researchers first looked for genetic abnormalities in FOLR1, the gene that codes for FR $\alpha$, but no genetic abnormalities were found. Next, the Quadros group at his laboratory at the State University of New York found autoantibodies to the FR $\alpha$ in CFD patients that interfered with the function of FR $\alpha$ [36] (Figure 3). Two types of autoantibodies, binding and blocking, were described. The blocking autoantibody was found to block the ability of folate to attach to FR $\alpha$ while the binding autoantibody recognizes an epitope distant from the folate-binding site where it binds and interferes with FR $\alpha$ function. Interestingly, bovine milk contains the FR $\alpha$ and eliminating cow's milk has been found to markedly reduce FR $\alpha$ autoantibody (FRAAs) titers with re-exposure to cow's milk significantly increasing titers [37]. These data suggest that immune cells in the gut may be reacting to the FR $\alpha$ in ingested milk to produce FRAAs. Since the identification of FRAAs, several case studies and series have linked metabolic disorders, particularly mitochondrial disease, to CFD as well [38].

\section{CFD in patients with ASDs}

Early reports found that some children with CFD also had ASD [39] with further studies suggesting that CFD was linked to children with low-functioning autism who also had neurological abnormalities [40]. Other studies documented CFD in a genetic disorder closely related to ASD, Rett syndrome [39,41]. About 5 years ago, we decided to determine the prevalence of FRAAs in children with idiopathic ASD. In our study, $75 \%$ of children with ASD were found to have at least one of the FRAAs with approximately $25 \%$ having both FRAAs [42]. Recently, studies in a separate population have confirmed this high FRAA prevalence in ASD [43,44]. Furthermore, Dr Vincent Ramaekers in Belgium has found that $60 \%$ of children with ASD were positive for the blocking FRAA with only $3 \%$ of developmentally delayed non-ASD controls being positive for the blocking FRAA [45]. Additionally, mitochondrial dysfunction, with or without FRAAs, is associated with ASD [46] and we have described children with ASD and mitochondrial disorders who have CFD [47]. This suggests that even in the absence of FRAAs, if there is abnormal mitochondrial function, folate transport into the CNS may be compromised. Given that folate 
supplementation was recently shown to improve mitochondrial function [25], this could be clinically important in patients that have markers of mitochondrial dysfunction.

\section{Folinic acid treatment in children with ASD}

One of the most important aspects of the discovery of CFD is that it is a potentially treatable condition. Specifically, a special type of reduced folate known as folinic acid (aka leucovorin calcium) was found to reverse neurologic and developmental abnormalities in many of the children diagnosed with CFD when given at high doses. In fact, some children with ASD were found to recover completely from their symptoms [48]. It is believed that, being a reduced folate, folinic acid can be transported through the RFC if the FR $\alpha$ is blocked (i.e., due to FRAAs) or nonfunctional (e.g., through mitochondrial dysfunction or genetic abnormalities). Since the RFC has a lower affinity for folate as compared with the $\mathrm{FR} \alpha$, the blood folate concentration needs to be relatively higher to optimize this alternative pathway. Furthermore, the average CSF folate concentration in children is about $80 \mathrm{nmol} / \mathrm{l}$ with the lower end of the normal range being about $40 \mathrm{nmol} / \mathrm{l}$. In comparison, the serum (peripheral) blood folate concentration in children is much lower with a range from about 10 to $45 \mathrm{nmol} / 1$. Therefore, there is steep concentration gradient for folate across the blood-brain barrier, highlighting the importance of an efficient transport of folate into the brain [36].

In a recent open-label study, 44 FRAA-positive children with ASD were treated for an average of 4 months with high-dose folinic acid $(2 \mathrm{mg} / \mathrm{kg} /$ day in two divided doses; maximum $50 \mathrm{mg} /$ day). Treatment response was based on the parents completing a 7-point Likert scale of improvement/worsening on nine aspects of behavior and development. These ratings were compared with 9 FRAA-positive ASD children in a wait-list control group who did not change treatment during the observation period. Overall, about two-thirds of those treated reported benefits and about a third reported marked improvements. Statistical analysis demonstrated greater improvements in expressive and receptive language, verbal communication, attention and stereotypic behavior in those treated as compared with the wait-list control group [42].

More recently, a double-blind placebo-controlled randomized trial treated children with ASD and language delay with high-dose folinic acid (or placebo) for 12 weeks. Children treated with the high-dose folinic acid showed significant improvements in core ASD symptoms as compared to those treated with placebo [43]. FRAAs were measured at the beginning of the study to determine whether this biomarker could predict response to the folinic acid treatment. Verbal communication, the primary outcome, was found to improve significantly in those treated with folinic acid (leucovorin calcium) at $2 \mathrm{mg} / \mathrm{kg} /$ day in two divided doses (maximum $50 \mathrm{mg} /$ day) as compared with placebo. Also, FRAA-positive children were found to be more likely to respond to folinic acid as compared with FRAA-negative children. Additional outcome measures and analyses demonstrated improvements in other areas of behavior and development, including improvements in daily living skills, irritability, lethargy, stereotyped behavior, hyperactivity, inappropriate speech and internalizing problems. The safety of folinic acid was found to be excellent with no difference in reported adverse effects between folinic acid or placebo treated groups.

\section{Effect of FRAAs in pregnancy}

Rats on a folate-deficient diet frequently have embryos that resorbed in the first few days after embryonic development resulting in decreased fertility. This led us to investigate the effects of a polyclonal antiserum to the rat placental FRs on folate metabolism and fetal development. Antibody administration on gestational day 8 was dose dependent with embryonic malformations at lower doses and complete resorption of embryos at higher doses suggesting that antibody targeting the FRs in a pregnant rat had the same effects as severe folate deficiency. This initial observation led us to investigate women with a history of neural tube defect (NTD) pregnancy. This investigation confirmed the presence of these autoantibodies in women with a history of NTD pregnancies [49]. Prior to this discovery, the explanation for the benefits of folate supplementation was not evident because most women with NTD pregnancy do not have genetic mutations in the folate metabolism pathways or systemic folate deficiency. The discovery of FRAAs provided a potential mechanism by which fetal folate insufficiency could occur in the presence of normal folate status in the mother.

\section{Folate abnormalities in pregnancy may contribute to ASD}

Some of the same abnormalities in folate metabolism have been found in mothers of children with ASD. For example, the same polymorphism in the RFC that has been found in children with ASD is also present in the mothers of children with ASD [50] and is linked to DNA methylation changes in their children [50]. In addition, 
many mothers of ASD children positive for FRAAs are indeed themselves positive for FRAAs [42,45]. The notion that disruption of folate metabolism during gestation could contribute to ASD is supported by two epidemiological studies which demonstrated that folate supplementation during the preconception period as well as pregnancy was protective of offspring from developing ASD or NTD [51,52]. It has also been suggested that maternal microbiome disruption during pregnancy could also contribute to abnormal folate metabolism during pregnancy and be associated with ASD development [12].

The connection between FRAAs during pregnancy and ASD is supported by a recent rodent model developed in the Quadros laboratory. Rats exposed to rat FR antibodies (FRAs) during gestation and the preweaning period demonstrated learning, cognitive and behavioral impairments including behaviors analogous to stereotyped repetitive movements and anxiety [53]. In follow-up studies, these deficits were found to be prevented when folinic acid and/or dexamethasone were given, either in combination or in isolation, during gestation, thus demonstrating that the effect of FRAs could be surmounted during gestation and that the effect involved immune activation [54]. Further, in the rodent model, FRAs were found to bind to the placenta as well as the choroid plexus in the fetus and the FRAs were associated with a significant attenuation of folate transfer to the fetus [54]. Thus, these data suggest that in humans, FRAAs are significant and can reduce folate delivery to the fetus in general and the fetal CNS specifically.

Folate supplementation during pregnancy is almost a universal recommendation but this recommendation has been questioned recently, primarily due to an unpublished study that claimed a connection between high maternal blood folate concentrations and an increase in ASD risk. These findings have been interpreted without considering the folate metabolism abnormalities discussed above. For example, abnormal function of the FR $\alpha$ and RFC would reduce the entry of folate into the brain and into the fetus, potentially resulting in a greater concentration of folate in the blood, especially if folate is being supplemented. In this way, an elevated blood folate concentration may be a biomarker of abnormal folate metabolism and a sign that folate is not being transported into critical regions of the body.

\section{Folate metabolism: an example of environment-genome interaction}

There are several variations in key genes implicated in one-carbon folate metabolism associated pathways that have been disrupted in children with ASD and are also found in the mothers of children that develop ASD. Interestingly, James et al. found that the combination of several of these genetic variations were associated with ASD rather than a single genetic variation [11]. However, genetic defects do not appear to be the only factors at play. Indeed several environmental factors can significantly influence folate metabolism, including the FRAAs and microbiome disruption (Figure 4). These environmental factors no doubt can work in concert to further disrupt folate metabolism along with polymorphisms. These can promote complex metabolic and endophenotypic variations that may promote ASD formation in susceptible individuals.

Additionally, environmental factors, such as exposure to toxicants, are associated with ASD and can result in inflammation and oxidative stress which, in turn, can result in mitochondrial dysfunction and damage to DNA and RNA [5]. Such environmental factors combined with a reduced availability of folate and glutathione can worsen mitochondrial function, cause methylation abnormalities and neurotransmitter depletion and limit the ability of the cells to repair damaged DNA.

Abnormalities related to DNA replication may be more problematic for mitochondrial DNA (mtDNA) since mtDNA is particularly sensitive to environmental toxicants and oxidative stress. Mitochondria are consistently turning over and replicating in order to maintain optimal function. This turnover requires replication of mtDNA. In addition, during embryonic and fetal development, the 100s of mitochondria in each cell need to be replicated during cell division. A decreased folate pool has been linked to acquired mtDNA mutations [55,56]. This has the potential to affect a substantial number of developing cells if folate insufficiency occurs prenatally.

Indeed, as compared with their typically developing siblings, children with ASD are enriched in heteroplasmic mutations in nonpolymorphic mtDNA regions, suggesting that errors in mtDNA replication occurred early in life, potentially during gestation [57].

Several studies have now shown that many ASD cases with genetic etiologies are associated with rare de novo mutations that are acquired, not inherited, potentially as a result of secondary errors in DNA maintenance [58,59]. Other studies suggest that mutations in the majority of siblings with ASD are on different genes, again suggesting that siblings share errors in the process of DNA replication and maintenance rather than inheriting a common mutation [60]. One common factor shared with siblings is the prenatal-maternal environment, which is increasingly 


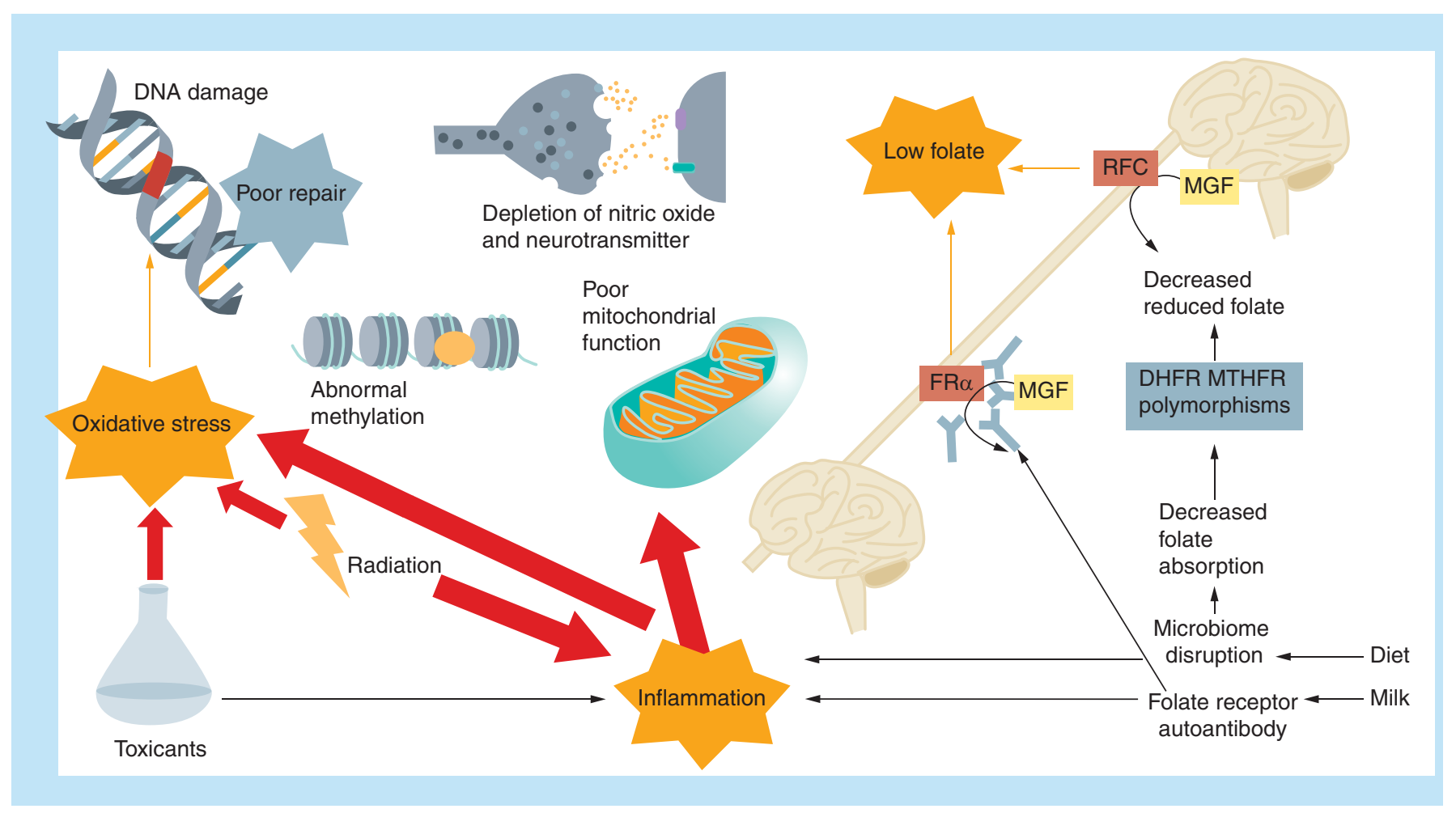

Figure 4. Interaction of environmental and predisposing factors in the folate pathways to result in neurological dysfunction. FR $\alpha$ : Folate receptor alpha; MGF: Monoglutamated folate; MTHFR: Methylenetetrahydrofolate reductase; RFC: Reduced folate carrier.

being recognized as important for developmental outcomes [61]. Thus, abnormalities in folate metabolism during gestation could explain such a relationship between de novo mutations and ASD.

\section{Conclusion}

Abnormalities in folate metabolism are one of the most prevalent metabolic abnormalities in children with ASD and their mothers. In this article we have pointed to the many biomarkers that can be used to detect these folate abnormalities. Specifically, clinical trials support the notion that FRAAs predict response to folinic acid (leucovorin calcium) in children with ASD. There are other known abnormalities in the folate pathway that are associated with ASD including polymorphisms in RFC, DHFR and MTHFR (Table 2) but these polymorphisms have not been studied in relation to treatment response or prevention. The folate pathways are connected to several other important pathways that are abnormal in ASD (Figure 2). Biomarkers in these pathways may also be useful in understanding ASD, detecting ASD early, diagnosing ASD, predicting treatment response and identifying ASD subtypes [62]. However, further research is needed.

\section{Future perspective}

What is promising is that the treatment with the appropriate type of reduced folate has the potential to reduce symptoms in children with ASD and potentially prevent ASD in the offspring if mothers with these same abnormalities in folate metabolism are identified and treated appropriately. Insufficiency in folate may also account for other abnormalities commonly associated with ASD. For example, mitochondrial dysfunction [25] has been shown to be highly prevalent in children with ASD [46], including biomarkers of mitochondrial dysfunction that have been found in the brain $[63,64]$. Interestingly mitochondrial dysfunction does not appear to have a clear genetic basis in ASD [46]. Folate is essential for mitochondrial function [25,65], raising the possibility that insufficiency of folate could account for mitochondrial abnormalities. Others have recently suggested that folate abnormalities and ASD may be connected through other metabolic pathways that are commonly abnormal to both such as serine, lysine and/or purine metabolism [66]. We have recently demonstrated that FRAAs during gestation may play a role in thyroid abnormalities present in children with ASD [67]. Furthermore, genetic syndromes associated with ASD 
such as Rett syndrome [68-70] and Down syndrome [71,72] demonstrate abnormalities in folate metabolism. Thus, a better understanding of how to identify and treat folate pathway abnormalities could have a significant impact in ASD and other neurodevelopmental disorders.

Understanding and treating folate abnormalities in ASD shows great promise for both treating symptoms and potentially for preventing ASD from occurring. However, there are many questions that need to be answered in order to provide effective treatment recommendations for more people affected by ASD or at risk for having a child with ASD. The role of FRAAs and other folate pathway abnormalities need to be better understood in children with ASD and their mothers. This can enable us to determine how much folate pathway abnormalities overlap with other abnormalities associated with ASD and will allow the development of specific biomarkers. The optimal treatment of folate pathway abnormalities needs to be investigated further in order to determine the specific type and dose of folate to be used and whether concurrent treatment with other cofactors is useful. Clearly, folates have the potential for a relatively safe and well-tolerated treatment for children with ASD where few other treatments are available.

Financial \& competing interests disclosure

This research was supported, in part, by a basic science research grant \# 8202 from Autism Speaks to EV Quadros and clinical research funding from the Autism Research Institute, the Arkansas Biosciences Institute, Brenen Hornstein Autism Research and Education Foundation, the Fraternal Order of Eagles of Arkansas, the Jane Botsford Johnson Foundation and Lee Silsby Compounding Pharmacy to RE Frye. RE Frye and EV Quadros are on the Scientific Advisory Board of Illiad Neurosciences Inc. (Plymouth Meeting, PA). EV Quadros is the inventor on a US patent no. for the detection of FR autoantibodies issued to the Research Foundation of the State University of New York. The authors have no other relevant affiliations or financial involvement with any organization or entity with a financial interest in or financial conflict with the subject matter or materials discussed in the manuscript apart from those disclosed.

No writing assistance was utilized in the production of this manuscript.

\section{Open access}

This work is licensed under the Attribution-NonCommercial-NoDerivatives 4.0 Unported License. To view a copy of this license, visit http://creativecommons.org/licenses/by-nc-nd/4.0/

\section{Executive summary}

Folate, one of the body's most important vitamins

- Folate (Vitamin B9) is important for many physiological systems that are critically involved in growth and development starting at conception, including gene replication and regulation, methylation, neurotransmitter production and redox metabolism.

Dietary folate \& the microbiome

- Factors within the gastrointestinal lumen, such as $\mathrm{pH}$ and aberrant microbiota may influence the amount and type of folate absorbed from the diet.

Autism spectrum disorder \& folate metabolism

- Autism spectrum disorder (ASD), a neurodevelopmental disorder that has increased in prevalence, has been linked to multiple abnormalities in folate metabolism.

- Polymorphisms in genes that encode enzymes in the folate and closely related pathways interact to increase the risk of developing ASD.

The folate receptor- $\alpha$ is important in neurodevelopmental disorders

- The folate receptor alpha $(F R \alpha)$ is part of a mechanism that actively transports folate into several body organs including the fetus and the brain.

- Autoantibodies have been identified which interfere with the ability of the FR $\alpha$ to function. These autoantibodies are highly prevalent in children with ASD.

Folate abnormalities can be treated in ASD

- Folinic acid (also known as leucovorin calcium), a reduced form of folate, may be an effective treatment for children with ASD and FR $\alpha$ autoantibodies.

Folate metabolism abnormalities also affect mothers of children with autism

- Mothers of children with ASD have similar abnormalities in folate metabolism as their children.

- Folate supplementation during the preconception and gestational period may be able to prevent the development of ASD. 


\section{References}

Papers of special note have been highlighted as: $\bullet \bullet$ of considerable interest

1. Zablotsky B, Black LI, Maenner MJ, Schieve LA, Blumberg SJ. Estimated prevalence of autism and other developmental disabilities following questionnaire changes in the 2014 National Health Interview Survey. Natl Health Stat. Rep. 87, 1-20 (2015).

2. Frye RE, Rossignol DA. Identification and treatment of pathophysiological comorbidities of autism spectrum disorder to achieve optimal outcomes. Clin. Med. Insights Pediatr. 10, 43-56 (2016).

3. Rossignol DA, Frye RE. A review of research trends in physiological abnormalities in autism spectrum disorders: immune dysregulation, inflammation, oxidative stress, mitochondrial dysfunction and environmental toxicant exposures. Mol. Psychiatry 17(4), 389-401 (2012).

4. Tammimies K, Marshall CR, Walker S et al. Molecular diagnostic yield of chromosomal microarray analysis and whole-exome sequencing in children with autism spectrum disorder. JAMA 314(9), 895-903 (2015).

5. Rossignol DA, Genuis SJ, Frye RE. Environmental toxicants and autism spectrum disorders: a systematic review. Transl. Psychiatry 4 , e360 (2014).

-• One of the most comprehensive reviews on the evidence for toxicant association with autism.

6. Hallmayer J, Cleveland S, Torres A et al. Genetic heritability and shared environmental factors among twin pairs with autism. Arch. Gen. Psychiatry 68(11), 1095-1102 (2011).

7. Sandin S, Lichtenstein P, Kuja-Halkola R, Larsson H, Hultman CM, Reichenberg A. The familial risk of autism. JAMA 311(17), 1770-1777 (2014).

8. Reichow B, Barton EE, Boyd BA, Hume K. Early intensive behavioral intervention (EIBI) for young children with autism spectrum disorders (ASD). Cochrane Database Syst. Rev. 10, CD009260 (2012).

9. Board IOMFaN. Dietary Reference Intakes: Thiamin, Riboflavin, Niacin, Vitamin B6, Folate, Vitamin B12, Pantothenic Acid, Biotin, and Choline. National Academy Press, DC, USA (1998).

10. Attwood SE, Ell C, Galmiche JP et al. Long-term safety of proton pump inhibitor therapy assessed under controlled, randomised clinical trial conditions: data from the SOPRAN and LOTUS studies. Aliment. Pharmacol. Ther. 41(11), 1162-1174 (2015).

11. James SJ, Melnyk S, Jernigan S et al. Metabolic endophenotype and related genotypes are associated with oxidative stress in children with autism. Am. J. Med. Genet. B Neuropsychiatr. Genet. 141B(8), 947-956 (2006).

-• Landmark paper associating increased autism risk with interactions between several polymorphisms in the folate and related pathways.

12. Slattery J, Macfabe DF, Kahler SG, Frye RE. Enteric ecosystem disruption in autism spectrum disorder: can the microbiota and macrobiota be restored? Curr. Pharm. Des. 22(40), 6107-6121 (2016).

13. Sugahara H, Odamaki T, Hashikura N, Abe F, Xiao JZ. Differences in folate production by bifidobacteria of different origins. Biosci. Microbiota Food Health 34(4), 87-93 (2015).

14. D'aimmo MR, Modesto M, Mattarelli P, Biavati B, Andlid T. Biosynthesis and cellular content of folate in bifidobacteria across host species with different diets. Anaerobe 30, 169-177 (2014).

15. D'aimmo MR, Mattarelli P, Biavati B, Carlsson NG, Andlid T. The potential of bifidobacteria as a source of natural folate. J. Appli. Microbiol. 112(5), 975-984 (2012).

16. Kang DW, Park JG, Ilhan ZE et al. Reduced incidence of Prevotella and other fermenters in intestinal microflora of autistic children. PLoS ONE 8(7), e68322 (2013).

17. Rossi M, Amaretti A, Raimondi S. Folate production by probiotic bacteria. Nutrients 3(1), 118-134 (2011).

18. Slattery J, Macfabe DF, Frye RE. The significance of the enteric microbiome on the development of childhood disease: a review of prebiotic and probiotic therapies in disorders of childhood. Clin. Med. Insights Pediatr. 10, 91-107 (2016).

19. Adams M, Lucock M, Stuart J, Fardell S, Baker K, Ng X. Preliminary evidence for involvement of the folate gene polymorphism 19bp deletion-DHFR in occurrence of autism. Neurosci. Lett. 422(1), 24-29 (2007).

20. Frustaci A, Neri M, Cesario A et al. Oxidative stress-related biomarkers in autism: systematic review and meta-analyses. Free Radic. Biol. Med. 52(10), 2128-2141 (2012).

21. Desai A, Sequeira JM, Quadros EV. The metabolic basis for developmental disorders due to defective folate transport. Biochimie 126, 31-42 (2016).

22. Pogribny IP, Tryndyak VP, Ross SA, Beland FA. Differential expression of microRNAs during hepatocarcinogenesis induced by methyl deficiency in rats. Nutr. Rev. 66(Suppl. 1), S33-S35 (2008).

23. Frye RE, Huffman LC, Elliott GR. Tetrahydrobiopterin as a novel therapeutic intervention for autism. Neurotherapeutics 7(3), 241-249 (2010).

24. Frye RE, Delatorre R, Taylor HB et al. Metabolic effects of sapropterin treatment in autism spectrum disorder: a preliminary study. Transl. Psychiatry 3, e237 (2013).

25. Delhey LM, Nur Kilinc E, Yin L et al. The effect of mitochondrial supplements on mitochondrial activity in children with autism spectrum disorder. J. Clin. Med. 6(2), pii:E18 (2017). 
26. Frye RE, James SJ. Metabolic pathology of autism in relation to redox metabolism. Biomark. Med. 8(3), 321-330 (2014).

27. Frye RE, Melnyk S, Fuchs G et al. Effectiveness of methylcobalamin and folinic acid treatment on adaptive behavior in children with autistic disorder is related to glutathione redox status. Autism Res. Treat. 2013, 609705 (2013).

28. Berrocal-Zaragoza MI, Sequeira JM, Murphy MM et al. Folate deficiency in rat pups during weaning causes learning and memory deficits. Br. J. Nutr. 112(8), 1323-1332 (2014).

29. Blaise SA, Nedelec E, Schroeder H et al. Gestational vitamin B deficiency leads to homocysteine-associated brain apoptosis and alters neurobehavioral development in rats. Am. J. Pathol. 170(2), 667-679 (2007).

30. Craciunescu CN, Albright CD, Mar MH, Song J, Zeisel SH. Choline availability during embryonic development alters progenitor cell mitosis in developing mouse hippocampus. J. Nutr. 133(11), 3614-3618 (2003).

31. Xiao S, Hansen DK, Horsley ET et al. Maternal folate deficiency results in selective upregulation of folate receptors and heterogeneous nuclear ribonucleoprotein-E1 associated with multiple subtle aberrations in fetal tissues. Birth Defects Res. Part A Clin. Mol. Teratol. 73(1), 6-28 (2005).

32. Ferguson SA, Berry KJ, Hansen DK, Wall KS, White G, Antony AC. Behavioral effects of prenatal folate deficiency in mice. Birth Defects Res. Part A Clin. Mol. Teratol. 73(4), 249-252 (2005).

33. Kim JM, Lee H, Chang N. Hyperhomocysteinemia due to short-term folate deprivation is related to electron microscopic changes in the rat brain. J. Nutr. 132(11), 3418-3421 (2002).

34. Iskandar BJ, Rizk E, Meier B et al. Folate regulation of axonal regeneration in the rodent central nervous system through DNA methylation. J. Clin. Invest. 120(5), 1603-1616 (2010).

35. Ramaekers VT, Blau N. Cerebral folate deficiency. Dev. Med. Child Neurol. 46(12), 843-851 (2004).

36. Ramaekers VT, Rothenberg SP, Sequeira JM et al. Autoantibodies to folate receptors in the cerebral folate deficiency syndrome. N. Engl. J. Med. 352(19), 1985-1991 (2005).

37. Ramaekers VT, Sequeira JM, Blau N, Quadros EV. A milk-free diet downregulates folate receptor autoimmunity in cerebral folate deficiency syndrome. Dev. Med. Child Neurol. 50(5), 346-352 (2008).

38. Garcia-Cazorla A, Quadros EV, Nascimento A et al. Mitochondrial diseases associated with cerebral folate deficiency. Neurology 70(16), 1360-1362 (2008).

39. Ramaekers VT, Hansen SI, Holm J et al. Reduced folate transport to the CNS in female Rett patients. Neurology 61(4), 506-515 (2003).

40. Ramaekers VT, Blau N, Sequeira JM, Nassogne MC, Quadros EV. Folate receptor autoimmunity and cerebral folate deficiency in low-functioning autism with neurological deficits. Neuropediatrics 38(6), 276-281 (2007).

41. Ramaekers VT, Sequeira JM, Artuch R et al. Folate receptor autoantibodies and spinal fluid 5-methyltetrahydrofolate deficiency in Rett syndrome. Neuropediatrics 38(4), 179-183 (2007).

42. Frye RE, Sequeira JM, Quadros EV, James SJ, Rossignol DA. Cerebral folate receptor autoantibodies in autism spectrum disorder. Mol. Psychiatry 18(3), 369-381 (2013).

-. The first systematic treatment study of folinic acid in children with autism and the folate receptor alpha autoantibody.

43. Frye RE, Slattery J, Delhey L et al. Folinic acid improves verbal communication in children with autism and language impairment: a randomized double-blind placebo-controlled trial. Mol. Psychiatry doi:10.1038/mp.2016.168 (2016) (Epub ahead of print).

-• The double-blind placebo-controlled study demonstrated the positive effect of folinic acid on verbal communication in children with autism.

44. Frye RE, Delhey L, Slattery J et al. Blocking and binding folate receptor alpha autoantibodies identify novel autism spectrum disorder subgroups. Front. Neurosci 10, 80 (2016).

45. Ramaekers VT, Quadros EV, Sequeira JM. Role of folate receptor autoantibodies in infantile autism. Mol. Psychiatry 18(3), 270-271 (2013).

46. Rossignol DA, Frye RE. Mitochondrial dysfunction in autism spectrum disorders: a systematic review and meta-analysis. Mol. Psychiatry 17(3), 290-314 (2012).

47. Frye RE, Naviaux RK. Autistic disorder with complex IV overactivity: a new mitochondrial syndrome. J. Ped. Neuro 9(4), 427-434 (2011).

48. Moretti P, Sahoo T, Hyland K et al. Cerebral folate deficiency with developmental delay, autism, and response to folinic acid. Neurology 64(6), 1088-1090 (2005).

49. Rothenberg SP, Da Costa MP, Sequeira JM et al. Autoantibodies against folate receptors in women with a pregnancy complicated by a neural-tube defect. N. Engl. J. Med. 350(2), 134-142 (2004).

50. James SJ, Melnyk S, Jernigan S et al. A functional polymorphism in the reduced folate carrier gene and DNA hypomethylation in mothers of children with autism. Am. J. Med. Genet. B Neuropsychiatr. Genet. 153B(6), 1209-1220 (2010).

51. Suren P, Roth C, Bresnahan M et al. Association between maternal use of folic acid supplements and risk of autism spectrum disorders in children. JAMA 309(6), 570-577 (2013). 
52. Schmidt RJ, Tancredi DJ, Ozonoff S et al. Maternal periconceptional folic acid intake and risk of autism spectrum disorders and developmental delay in the CHARGE (CHildhood Autism Risks from Genetics and Environment) case-control study. Am. J. Clin. Nutr. 96(1), 80-89 (2012).

53. Sequeira JM, Desai A, Berrocal-Zaragoza MI, Murphy MM, Fernandez-Ballart JD, Quadros EV. Exposure to folate receptor alpha antibodies during gestation and weaning leads to severe behavioral deficits in rats: a pilot study. PLoS ONE 11(3), e0152249 (2016).

54. Desai A, Sequeira JM, Quadros EV. Prevention of behavioral deficits in rats exposed to folate receptor antibodies: implication in autism. Mol. Psychiatry doi:10.1038/mp.2016.153 (2016) (Epub ahead of print).

-. Landmark study demonstrating that the animal models of the folate receptor autoantibody can be rescued with folate and/or steroid treatment, thereby supporting the clinical evidence in children with autism.

55. Chang CM, Yu CC, Lu HT, Chou YF, Huang RF. Folate deprivation promotes mitochondrial oxidative decay: DNA large deletions, cytochrome c oxidase dysfunction, membrane depolarization and superoxide overproduction in rat liver. Br. J. Nutr. 97(5), 855-863 (2007).

56. Chou YF, Huang RF. Mitochondrial DNA deletions of blood lymphocytes as genetic markers of low folate-related mitochondrial genotoxicity in peripheral tissues. Eur. J. Nutr. 48(7), 429-436 (2009).

57. Wang Y, Picard M, Gu Z. Genetic evidence for elevated pathogenicity of mitochondrial DNA heteroplasmy in autism spectrum disorder. PLoS Genet. 12(10), e1006391 (2016).

58. Neale BM, Kou Y, Liu L et al. Patterns and rates of exonic de novo mutations in autism spectrum disorders. Nature 485(7397), 242-245 (2012).

59. Sanders SJ, Murtha MT, Gupta AR et al. De novo mutations revealed by whole-exome sequencing are strongly associated with autism. Nature 485(7397), 237-241 (2012).

60. Yuen RK, Thiruvahindrapuram B, Merico D et al. Whole-genome sequencing of quartet families with autism spectrum disorder. Nat. Med. 21(2), 185-191 (2015).

61. Adams JB, Pollard EL, Perez LM et al. The Science Behind the Healthy Child Guide. The Neurological Health Foundation, TX, USA (2016).

62. Howsmon DP, Kruger U, Melnyk S, James SJ, Hahn J. Classification and adaptive behavior prediction of children with autism spectrum disorder based upon multivariate data analysis of markers of oxidative stress and DNA methylation. PLoS Comput. Biol. 13(3), e1005385 (2017).

63. Goh S, Dong Z, Zhang Y, Dimauro S, Peterson BS. Mitochondrial dysfunction as a neurobiological subtype of autism spectrum disorder: evidence from brain imaging. JAMA Psychiatry 71(6), 665-671 (2014).

64. Rossignol DA, Frye RE. Evidence linking oxidative stress, mitochondrial dysfunction, and inflammation in the brain of individuals with autism. Front. Physiol. 5, 150 (2014).

65. Niyazov DM, Kahler SG, Frye RE. Primary mitochondrial disease and secondary mitochondrial dysfunction: importance of distinction for diagnosis and treatment. Mol. Syndromol. 7(3), 122-137 (2016).

66. Krsicka D, Geryk J, Vlckova M, Havlovicova M, Macek M Jr, Pourova R. Identification of likely associations between cerebral folate deficiency and complex genetic- and metabolic pathogenesis of autism spectrum disorders by utilization of a pilot interaction modeling approach. Autism. Res. doi:10.1002/aur.1780 (2017) (Epub ahead of print).

67. Frye RE, Wynne R, Rose $\mathrm{S}$ et al. Thyroid dysfunction in children with autism spectrum disorder is associated with folate receptor alpha autoimmune disorder. J. Neuroendocrinol. 29(3), doi:10.1111/jne.12461 (2017) (Epub ahead of print).

68. Grosser E, Hirt U, Janc OA et al. Oxidative burden and mitochondrial dysfunction in a mouse model of Rett syndrome. Neurobiol. Dis. 48(1), 102-114 (2012).

69. Gibson JH, Slobedman B, K NH et al. Downstream targets of methyl CpG binding protein 2 and their abnormal expression in the frontal cortex of the human Rett syndrome brain. BMC Neurosci. 11, 53 (2010).

70. Condie J, Goldstein J, Wainwright MS. Acquired microcephaly, regression of milestones, mitochondrial dysfunction, and episodic rigidity in a 46,XY male with a de novo MECP2 gene mutation. J. Child Neurol. 25(5), 633-636 (2010).

71. Pagano G, Castello G. Oxidative stress and mitochondrial dysfunction in Down syndrome. Adv. Exp. Med. Biol. 724, 291-299 (2012).

72. Pallardo FV, Lloret A, Lebel $\mathrm{M}$ et al. Mitochondrial dysfunction in some oxidative stress-related genetic diseases: ataxia-telangiectasia, Down syndrome, Fanconi anaemia and Werner syndrome. Biogerontology 11(4), 401-419 (2010). 